\title{
Technical and Economic Efficiency Measures Under Short Run Profit Maximizing Behavior
}

\author{
Laurens Cherchye* \\ Timo Kuosmanen ${ }^{* *}$ \\ Hervé Leleu ${ }^{* * *}$
}

\section{Introduction}

Technology distance functions (Shephard, 1953, 1970; Chambers et al. $1996,1998)$ are used to measure the distance from a production plan in the interior of the production set to the boundary of that set. Such distance functions have proven particularly useful for technical efficiency measurement (following Farrell, 1957). Within that perspective, the distance function may theoretically adopt a multitude of directions of measurement as, in principle, any point on the boundary of the production set is an equally valid technically efficient reference point. For selecting the optimal direction, the efficiency analyst typically uses additional information regarding the behavioral objectives of the evaluated firm. If full price information is available, such behavioral assumptions entail economic efficiency analysis through the cost, revenue or profit function. The relationship between technical and economic efficiency relates to the absence of such price information: it is then natural to ask which technical (quantity based) efficiency measure offers a natural dual counterpart to the economic (price based) effi-

University of Leuven, Campus Kortijk and Center for Economic Studies; Fund for Scientific Research - Flanders (FWO-Vlaanderen). E. Sabbelaan 53, B-8500 Kortrijk, Belgium. E-mail: laurens.cherchye @ kulak.ac.be.

* Economic Research Unit, MTT Agrifood research Finland, Luutnantintie 13, 00410 Helsinki, Finland. E-mail: Timo.Kuosmanen@mtt.fi. Helsinki School of Economics, P.O. Box 1210, 00101 Helsinki, Finland. E-mail: Timo.Kuosmanen@hse.fi.

*** CNRS/LEM and IÉSEG School of Management, 3 rue de la Digue, F-59000 Lille, France, h.leleu @ ieseg.fr. Corresponding author. 
ciency measures. Russell (1985; p. 124) insightfully addressed this question as follows:

"Even if market prices are not known, however, economic efficiency is not irrelevant to the analysis of technical efficiency. (Both Debreu and Farrell emphasized the relationships between technical and economic efficiency.) In fact, even if market prices do not exist, notions of economic efficiency are not irrelevant to the analysis of technical efficiency: shadow prices, implicit in all production technologies are relevant."

In the case of cost-minimizing behavior, where the ratio of minimum to actual input costs is the usual measure of economic efficiency, Russell (1985) advocated the radial Shephard input distance function, the reciprocal of the Farrell (1957) efficiency measure, as the natural (quantity based) dual to this economic (cost efficiency) measure. ${ }^{1}$ He claimed two attractive properties of this distance function that are not shared by any other technical efficiency measure. First, while the economic efficiency is a ratio of minimal to actual cost at market prices, the input distance function can (dually) be interpreted as the ratio of the minimal to the actual cost at shadow prices (determined at a given level of output and in radial input direction). Second, the input distance function provides an upper bound for economic efficiency (using market prices).

Over the last few years, the general directional distance function has become increasingly popular in the literature on technical efficiency analysis. Chambers et al. $(1996,1998)$ originally developed it as a generalization of Luenberger's (1992) shortage function. An attractive property is that it allows for simultaneous variation of inputs and outputs, whereas the traditional distance functions consider either input or output fixed. Another appealing feature of the directional distance function is its generality; all known technical efficiency measures, including the original Farrell measures, can be expressed as special cases of the directional distance function through appropriate specification of the direction vector. However, this generality of the directional distance function simultaneously implies its main weakness. In empirical application, the efficiency results (including efficiency values and rankings) will crucially depend on the choice of the direction vector (which determines the projection direction towards the technically efficient frontier). Unfortunately, the literature of directional distance functions does not provide any guidance as to the specification of the direction vector. Moreover, Salnykov and Zeleniuk (2005) show that a desirable property such as the commensurability (independence of units of measurement up to a scalar transformation) is not systematically satisfied by the directional distance function.

1 Russell specifically referred to the duality between the cost function and Shephard's input distance function. This special relationship between radial technical efficiency measures and economic efficiency was well understood by Farrell (1957) when originally introducing these measures in the context of productive efficiency analysis. 
This paper provides a dual perspective on the issue of specifying the direction vector. Following Russell (1985), we identify the direction that entails a (quantity based) directional distance function that has a naturally dual interpretation in terms of economic efficiency. Of course, this first requires a specification of the appropriate economic efficiency notion, which in turn refers to specific behavioral assumptions. Given that the directional distance function encompasses simultaneous changes in both inputs and outputs, we start from profit maximization as the appropriate behavioral assumption, which leads to profit efficiency as the economic efficiency notion.

The measurement of firm profit efficiency dates back to at least Nerlove (1965), who presented two alternative efficiency indices for that purpose. Following Nerlove, we focus on the usually relevant notion of short run profit efficiency, which incorporates the managerial constraint that some inputs (e.g. capital stock) are to be considered as fixed within the time-frame of the analysis. In the following, we adapt alternative efficiency notions, including Varian's (1990) goodness of fit measure and Chambers et al.'s (1998) generalization of the Nerlovian profit efficiency, to this short run perspective by distinguishing between fixed and variable inputs.

Building on the duality results of Chambers et al. (1998) and Färe and Grosskopf (2000), we then show that McFadden's (1978) gauge function provides a natural measurement direction in the context of short run profit efficiency. We claim that the corresponding specification of the direction vector obtains the same two properties that made Russell advocate the Shephard distance function within the cost efficiency setting, but now these properties apply for profit efficiency. Finally, to strengthen the case of the corresponding direction of measurement, we make explicit that the McFadden procedure selects a (technically efficient) reference production plan that may be interpreted as the "most favorable" from the evaluated firm's point of view.

The rest of the paper is organized as follows. The next section considers short run profit efficiency measurement. Section 3 institutes the McFadden gauge function as a naturally dual technical efficiency measure for profit efficiency as the economic objective. Section 4 summarizes our findings.

\section{Profit efficiency in the short run}

Following Nerlove (1965), we focus on the firm that is profit maximizing in the short run. We denote the (non-negative and non-zero) input vector by $\mathbf{x}$ and by $\mathbf{w}$ the corresponding price vector. The input vector is further divided into a subvector with fixed inputs $\mathbf{x}^{f}$ and one with variable inputs $\mathbf{x}^{v}$, which obtains $\mathbf{x}=\left(\mathbf{x}^{f}, \mathbf{x}^{v}\right)$. The (non-negative and non-zero) output vector 
is denoted by $\mathbf{y}$ and $\mathbf{p}$ is the output price vector. In the short run, the firm takes the level of fixed inputs as given and can only adjust the consumption of variable inputs. ${ }^{2}$ The firm's economic objective is then defined as the gross profit function

$$
\pi\left(\mathbf{x}^{f} ; \mathbf{w}, \mathbf{p}\right)=\sup _{\mathbf{x}^{v}, \mathbf{y}}\left\{\mathbf{p}^{\prime} \mathbf{y}-\mathbf{w}^{\prime} \mathbf{x}^{v} \mid\left(\mathbf{x}^{f} ; \mathbf{x}^{v}, \mathbf{y}\right) \in T\right\}
$$

where $T=\{(\mathbf{x}, \mathbf{y}) \mid \mathbf{x}$ can produce $\mathbf{y}\}$ is a production possibility set that is assumed to satisfy free disposability of input and output. We also assume that $\forall\left(\mathbf{x}^{\mathbf{f}} ; \mathbf{x}^{\mathbf{v}}, \mathbf{y}\right) \in T$ there is some $\delta>0$ such that $\left(\mathbf{x}^{\mathbf{f}} ; \delta \mathbf{x}^{\mathbf{v}}, \delta \mathbf{y}\right) \notin T$ (see Boussemart et al. (2008)). This assumption ensures that the maximum gross profit always exists and is finite.

For the given setting, we can distinguish at least four alternative profit efficiency measures (PEs) that have been suggested in the literature. Nerlove (1965) was the first to consider short run profit efficiency. He proposed two measures: a ratio measure

$$
P E^{R}\left(\mathbf{x}_{0}, \mathbf{y}_{0} ; \mathbf{w}_{0}, \mathbf{p}_{0}\right)=\frac{\pi\left(\mathbf{x}_{0}^{f} ; \mathbf{w}_{0}, \mathbf{p}_{0}\right)}{\mathbf{p}_{0}^{\prime} \mathbf{y}_{0}-\mathbf{w}_{0}^{\prime} \mathbf{x}_{0}^{v}}
$$

as well as an additive measure

$$
P E^{A}\left(\mathbf{x}_{0}, \mathbf{y}_{0} ; \mathbf{w}_{0}, \mathbf{p}_{0}\right)=\pi\left(\mathbf{x}_{0}^{f} ; \mathbf{w}_{0}, \mathbf{p}_{0}\right)-\left(\mathbf{p}_{0}^{\prime} \mathbf{y}_{0}-\mathbf{w}_{0}^{\prime} \mathbf{x}_{0}^{v}\right),
$$

where the subscript 0 refers to the observed prices/quantities of the evaluated firm. While the first PE evaluates profit efficiency in proportionate terms, the second one measures (absolute) profit loss due to inefficiency, expressed in money terms. ${ }^{3}$

Varian (1990) introduced a third alternative, which combines the previous two, namely ${ }^{4}$

$$
P E^{P}\left(\mathbf{x}_{0}, \mathbf{y}_{0} ; \mathbf{w}_{0}, \mathbf{p}_{0}\right)=\frac{\pi\left(\mathbf{x}_{0}^{f} ; \mathbf{w}_{0}, \mathbf{p}_{0}\right)-\left(\mathbf{p}_{0}^{\prime} \mathbf{y}_{0}-\mathbf{w}_{0}^{\prime} \mathbf{x}_{0}^{v}\right)}{\mathbf{p}_{0}^{\prime} \mathbf{y}_{0}-\mathbf{w}_{0}^{\prime} \mathbf{x}_{0}^{v}} \quad\left(=P E^{R}\left(\mathbf{x}_{0}, \mathbf{y}_{0} ; \mathbf{w}_{0}, \mathbf{p}_{0}\right)-1\right) .
$$

This PE can be interpreted as the percent extra profit that the firm would have generated at the given prices if it had used the optimal amounts of variable inputs to produce the optimal amounts of outputs.

2 Evidently, the identity of the 'fixed' inputs depends on the length of the planning horizon.

3 Mathematically, the ratio measure (1) is a direct analogue of Debreu's (1951) "coefficient of resource utilization". Debreu suggested that measure for investigating Pareto efficiency of the economy as a whole, and not for directly assessing firm profit efficiency. Yet, Debreu's coefficient has been widely interpreted as a cost efficiency ratio similar to that of Farrell (1957). However, Debreu's coefficient is defined in terms of net consumption in commodity space, where positive elements represent consumption while negative elements represent production; a dot product of the net consumption vector and the associated price vector then yields the net value of consumption. If we think of "the economy" as "a firm", then the produced commodities are the outputs while the consumed commodities are the inputs and, hence, the "net value of consumption" represents the "firm's profit".

4 Measure (3) adapts Varian's (1990) original goodness-of-fit measure to the current short run profit maximization setting. The same applies for measure (4). 
Finally, and most recently, Chambers et al. (1998) have presented a fourth, most general formulation of PE. Their approach is to normalize prices by introducing direction vectors $\mathbf{g}_{p}$ and $\mathbf{g}_{w}$, which yields

$$
P E^{D}\left(\mathbf{x}_{0}, \mathbf{y}_{0} ; \mathbf{w}_{0}, \mathbf{p}_{0} ; \mathbf{g}_{w}, \mathbf{g}_{p}\right)=\frac{\pi\left(\mathbf{x}_{0}^{f} ; \mathbf{w}_{0}, \mathbf{p}_{0}\right)-\left(\mathbf{p}_{0}^{\prime} \mathbf{y}_{0}-\mathbf{w}_{0}^{\prime} \mathbf{x}_{0}^{v}\right)}{\mathbf{p}_{0}^{\prime} \mathbf{g}_{p}+\mathbf{w}_{0}^{\prime} \mathbf{g}_{w}} .
$$

This general construction encompasses a multitude of PEs, depending on the specification of the direction vectors. For example, Varian's measure (3) is obtained for $\mathbf{g}_{\mathrm{p}}=\mathbf{y}_{0}$ and $\mathbf{g}_{\mathrm{w}}=-\mathbf{x}_{0}^{f}$.

It is interesting to look at the properties of the candidate measures (1)-(4). Let us first consider the ratio measures (1) and (3). An attractive property of these measures is that they are homogeneous of degree zero in prices and quantities (see also Nerlove, 1965), which makes them invariant to the units of measurement (such as the currency unit for the input and output prices). Still, they are generally ill-defined (i.e., they equal plus or minus infinity) if the observed profit equals zero. In the case of negative profits, the percentage measure has an advantage over the ratio measure in that the losses are revealed by the sign of the efficiency measure; this is not the case for the ratio measure if both the maximum profit and the observed profit are negative.

The opposite holds for the difference measure (2). This measure is no longer homogenous of degree zero in prices and quantities. But it is capable of handling negative or zero profits: its value is always a non-negative and finite real number interpretable as the absolute profit loss (in money terms).

Finally, the directional PE (4) combines the virtues of the measures (1)-(3): it is homogenous of degree zero in prices and quantities, and it is well-defined for zero profits. In fact, we believe these appealing features may (at least partly) explain the current popularity of this PE in the applied literature.

However, one major problem regarding the measure (4) concerns the choice of the normalization, and the concomitant economic interpretation. So far, the theoretical literature of directional distance functions has remained silent on this issue. Applications often select direction vectors $\mathbf{g}_{\mathrm{p}}=\mathbf{y}_{0}$ and $\mathbf{g}_{\mathrm{w}}=\mathbf{x}_{0}^{f}$, especially when the vector of variable inputs represents environmentally detrimental factors such as pollution or waste (e.g., Färe et al. 2001, Domazlicky and Weber, 2004). Still, in our opinion, this normalization lacks a sound economic interpretation. It entails that the profit loss in the numerator is proportioned to the sum of total revenue and total variable (or environmental) cost in the denominator. Since the total revenue is the sum of total variable cost and gross profit, this denominator actually equals the gross profit plus two times the total variable cost. Hence, direction $\mathbf{g}_{\mathrm{p}}=\mathbf{y}_{0}$ and $\mathbf{g}_{\mathrm{w}}=\mathbf{x}_{0}^{f}$ involves double counting of the total variable cost, which does not seem to have a good economic rationale. 
At this point, we recall that our focus is on short run profit maximizing firms. This complies with the original setting of Nerlove (1965), who considered gross profit (defined as the total revenue minus total variable cost), but deviates from Varian (1990) and Chambers et al. (1998), who either considered net profit (revenue minus total cost) or did not make an explicit distinction between the two notions. In practice, we may reasonably expect gross profit to be positive in the current (short run) set-up. Indeed, the corresponding variable costs merely include wages and material costs directly associated with the output, and they exclude all fixed costs as well as depreciation of capital, taxes, and other indirect costs included in the accounting profits and losses. Thus, negative or zero gross profit would imply that revenues do not suffice to pay the immediate production costs, which means that the firm owners would be better off by shutting down the enterprise; keeping up production under such conditions would require external equity or debt financing. We may thus abstract from non-positive gross profit in the short run.

In light of the above discussion, Varian's percentage measure with its attractive features appears as a most appropriate PE. In other words, we suggests $\mathbf{g}_{\mathrm{p}}=\mathbf{y}_{0}$ and $\mathbf{g}_{\mathrm{w}}=-\mathbf{x}_{0}^{f}$ as an economically meaningful normalization for the directional PE (4). ${ }^{5}$ (To avoid any possible confusion, we emphasize that our above argument applies for (short run) gross profit and not necessarily for net profit). The next section establishes the dual link between this specific economic efficiency measure and the (quantity based) McFadden gauge function.

\section{$3 \quad$ Technical efficiency}

Following Russell (1985), we ask for a quantity based technical efficiency measure (TE) that is dual to Varian's price based (gross) PE. Russell's conditions for such a TE are as follows: (i) the dual formulation of the TE should be interpretable as the PE at the input and output shadow price vectors; (ii) the TE should provide an upper bound for the PE at all prices. This section identifies the McFadden gauge as such a technical efficiency measure.

We start from the general directional distance function of Chambers et al. (1998)

$$
T E^{D}\left(\mathbf{x}, \mathbf{y} ; \mathbf{g}_{w}, \mathbf{g}_{p}\right)=\sup _{\delta \in R}\left\{\delta \mid\left(\mathbf{x}^{f}, \mathbf{x}^{v}-\delta \mathbf{g}_{w}, \mathbf{y}+\delta \mathbf{g}_{p}\right) \in T\right\} .
$$

5 We prefer Varian's PE (3) above the ratio PE (1) given its more natural percentage interpretation. Still, it should be clear that our below arguments for the PE (3) are easily adapted for the PE (1); see also their one-to-one correspondence that is made explicit in (3). 
In this $\mathrm{TE}$, the vectors $\mathbf{g}_{\mathrm{p}}$ and $\mathbf{g}_{\mathrm{w}}$ determine the direction of projection of the evaluated input-output vector onto the boundary of the production possibility set $T$. This general construction is the technical efficiency counterpart of the directional PE (4), which follows from the duality result (see Chambers et al., 1998) ${ }^{6}$

$$
\begin{aligned}
T E^{D}\left(\mathbf{x}_{0}, \mathbf{y}_{0} ; \mathbf{g}_{w}, \mathbf{g}_{p}\right) & =\sup _{\mathbf{w}, \mathbf{p} \geq \mathbf{0}} \frac{\pi\left(\mathbf{x}_{0}^{f} ; \mathbf{w}, \mathbf{p}\right)-\left(\mathbf{p}^{\prime} \mathbf{y}_{0}-\mathbf{w}^{\prime} \mathbf{x}_{0}^{v}\right)}{\mathbf{p}^{\prime} \mathbf{g}_{p}+\mathbf{w}^{\prime} \mathbf{g}_{w}} \\
& =P E^{D}\left(\mathbf{x}_{0}, \mathbf{y}_{0} ; \mathbf{w}^{*}, \mathbf{p}^{*} ; \mathbf{g}_{w}, \mathbf{g}_{p}\right),
\end{aligned}
$$

with $\left(\mathbf{w}^{*} \mathbf{p}^{*}\right.$ ) the (maximizing) shadow price vector given by the normal of the plane that supports the production set $T$ at the point $\left(\mathbf{x}_{0}^{f}, \mathbf{x}_{0}^{v}-\delta\left(\mathbf{x}_{0}, \mathbf{y}_{0} ; \mathbf{g}_{w}, \mathbf{g}_{p}\right) \mathbf{g}_{w}, \mathbf{y}+\delta\left(\mathbf{x}_{0}, \mathbf{y}_{0} ; \mathbf{g}_{w}, \mathbf{g}_{p}\right) \mathbf{g}_{p}\right)$. Comparing (6) to (3) obtains

$$
\begin{aligned}
T E^{D}\left(\mathbf{x}_{0}, \mathbf{y}_{0} ;-\mathbf{x}_{0}^{v}, \mathbf{y}\right) & =\sup _{\mathbf{p}, \mathbf{w} \geq 0} \frac{\pi\left(\mathbf{x}_{0}^{f} ; \mathbf{w}, \mathbf{p}\right)-\left(\mathbf{p}^{\prime} \mathbf{y}_{0}-\mathbf{w}^{\prime} \mathbf{x}_{0}^{v}\right)}{\mathbf{p}^{\prime} \mathbf{y}_{0}-\mathbf{w}^{\prime} \mathbf{x}_{0}^{v}} \\
& =P E^{P}\left(\mathbf{x}_{0}, \mathbf{y}_{0} ; \mathbf{w}^{*}, \mathbf{p}^{*}\right)
\end{aligned}
$$

We conclude that this TE calculates Varian's PE at shadow prices. We next establish the one-to-one correspondence between this TE and a variant of McFadden's (1978) gauge function defined as

$$
\operatorname{Mc} G\left(\mathbf{x}_{0}^{f} ; \mathbf{x}_{0}^{v}, \mathbf{y}_{0}\right)=\sup \left\{\delta:\left(\mathbf{x}_{0}^{f} ; \delta \mathbf{x}_{0}^{v}, \delta \mathbf{y}_{0}\right) \in T\right\}
$$

i.e., the McFadden gauge captures the maximal radial expansion of (simultaneously) the variable input and output vectors. The gauge function (8) can be interpreted as Shephard's output distance function with the variable inputs treated as outputs, and it thus inherits all properties of the latter. ${ }^{7}$ Like before (see footnote 4), we have adapted McFadden's original definition to the short run setting under consideration, by drawing a distinction between fixed and variable inputs. Besides improved consistency with the previous economic efficiency setting, the introduction of fixed inputs guarantees that the gauge function (8) always has a finite maximum, under the maintained assumption that the technology satisfies the "no free lunch" property. Hence, in contrast to the original McFadden gauge, which expands all inputs and outputs equiproportionately, our variant in (8) would remain well defined even if one allows the technology $T$ exhibit constant or increasing returns to scale.

6 This duality result requires that $\mathrm{T}$ satisfies convexity and monotonicity. If $\mathrm{T}$ is non-convex, the duality result applies for the technical efficiency measure defined relative to the convex monotonic hull of $T$ (see e.g. Kuosmanen, 2003, for details).

7 For a formal discussion of the properties of the output distance function, we refer to Färe and Primont (1995). 
The link between this gauge function and the TE (7) can be established as follows:

$$
\begin{aligned}
T E^{D}\left(\mathbf{x}_{0}, \mathbf{y}_{0} ;-\mathbf{x}_{0}^{v}, \mathbf{y}_{0}\right)= & \sup \left\{\delta \in R \mid\left(\mathbf{x}_{0}^{f} ; \mathbf{x}_{0}^{v}+\delta \mathbf{x}_{0}^{v}, \mathbf{y}_{0}+\delta \mathbf{y}_{0}\right) \in T\right\} \\
& =\sup \left\{\delta \in R \mid\left(\mathbf{x}_{0}^{f} ;(1+\delta) \mathbf{x}_{0}^{v},(1+\delta) \mathbf{y}_{0}\right) \in T\right\} \\
& =\sup \left\{(1+\delta) \in R \mid\left(\mathbf{x}_{0}^{f} ;(1+\delta) \mathbf{x}_{0}^{v},(1+\delta) \mathbf{y}_{0}\right) \in T\right\}-1 \\
& =\sup \left\{\delta:\left(\mathbf{x}_{0}^{f} ; \delta \mathbf{x}_{0}^{v}, \delta \mathbf{y}_{0}\right) \in T\right\}-1 \\
& =\operatorname{Mc} G\left(\mathbf{x}_{0}^{f} ; \mathbf{x}_{0}^{v}, \mathbf{y}_{0}\right)-1
\end{aligned}
$$

As a result, the McFadden gauge function reveals itself as a natural (quantity based) dual to the PE (3). Indeed, expression (6) makes explicit that it effectively complies with Russell's requirements (i) and (ii) cited above: its dual is interpretable as Varian's PE at the input and output shadow price vectors; and the TE provides an upper bound for that PE at all prices.

The same result can be also be interpreted from the perspective of the evaluated firm that has to choose the direction vector for the directional distance function used for its evaluation. Specifically, we obtain that the McFadden gauge function (8) implies the optimal (i.e., the "most favorable") direction vector from the evaluated firm's perspective: it selects a (technically efficient) reference production plan that minimizes the profit inefficiency as calculated with respect to the reference production plan.

To see this, we first note that in the general directional distance function framework the reference production plan for a given production plan $\left(\mathbf{x}_{0}^{f}, \mathbf{x}_{0}^{v}, \mathbf{y}_{0}\right)$ is

$$
\left(\mathbf{x}_{0}^{f}, \mathbf{x}_{0}^{v}-T E^{D}\left(\mathbf{x}_{0}, \mathbf{y}_{0} ; \mathbf{g}_{w}, \mathbf{g}_{p}\right) \mathbf{g}_{w}, \mathbf{y}_{0}+T E^{D}\left(\mathbf{x}_{0}, \mathbf{y}_{0} ; \mathbf{g}_{w}, \mathbf{g}_{p}\right) \mathbf{g}_{p}\right) .
$$

To simplify notation, we will further use $\mathbf{g}=\left(\mathbf{g}_{w}, \mathbf{g}_{p}\right)$. From (6), it follows that there should exists a shadow price system $\left(\mathbf{w}_{g}^{*} \mathbf{p}_{g}^{*}\right)$ that makes the technically efficient plan (10) short run profit maximizing, i.e.,

$\mathbf{p}_{g}^{*}\left(\mathbf{y}_{0}+T E^{D}\left(\mathbf{x}_{0}, \mathbf{y}_{0} ; \mathbf{g}_{w}, \mathbf{g}_{p}\right) \mathbf{g}_{p}\right)-\mathbf{w}_{g}^{*}\left(\mathbf{x}_{0}^{v}-T E^{D}\left(\mathbf{x}_{0}, \mathbf{y}_{0} ; \mathbf{g}_{w}, \mathbf{g}_{p}\right) \mathbf{g}_{w}\right)=\pi\left(\mathbf{x}_{0}^{f} ; \mathbf{w}_{g}^{*}, \mathbf{p}_{g}^{*}\right)$,

with $\pi\left(\mathbf{x}_{0}^{f} ; \mathbf{w}_{g}^{*}, \mathbf{p}_{g}^{*}\right)$ the (maximum) shadow profit level corresponding to the reference production plan. The PE value for the evaluated production plan is thus 


$$
\begin{gathered}
P E^{P}\left(\mathbf{x}_{0}, \mathbf{y}_{0} ; \mathbf{w}^{*}, \mathbf{p}^{*}\right)=\frac{\pi\left(\mathbf{x}_{0}^{f} ; \mathbf{w}_{g}^{*}, \mathbf{p}_{g}^{*}\right)-\left(\mathbf{p}_{g}^{*} \mathbf{y}_{0}-\mathbf{w}_{g}^{*} \mathbf{x}_{0}^{v}\right)}{\mathbf{p}_{g}^{*} \mathbf{y}_{0}-\mathbf{w}_{g}^{*} \mathbf{x}_{0}^{v}} \\
=\frac{\mathbf{p}_{g}^{*}\left(T E^{D}\left(\mathbf{x}_{0}, \mathbf{y}_{0} ; \mathbf{g}_{w}, \mathbf{g}_{p}\right) \mathbf{g}_{p}\right)+\mathbf{w}_{g}^{*}\left(T E^{D}\left(\mathbf{x}_{0}, \mathbf{y}_{0} ; \mathbf{g}_{w}, \mathbf{g}_{p}\right) \mathbf{g}_{w}\right)}{\mathbf{p}_{g}^{*} \mathbf{y}_{0}-\mathbf{w}_{g}^{*} \mathbf{x}_{0}^{v}} .
\end{gathered}
$$

The last equality makes clear that the profit efficiency generally depends on the choice of the direction and the associated optimal shadow prices. If the evaluated firm was given an opportunity to specify the direction vector $\mathbf{g}=\left(\mathbf{g}_{w}, \mathbf{g}_{p}\right)$, how would a rational firm choose the direction vector in this specific evaluation setting? The firm's problem is to minimize its profit inefficiency, so that the problem to solve becomes

$$
\inf _{\mathbf{g}}\left\{\frac{\mathbf{p}_{g}^{*}\left(T E^{D}\left(\mathbf{x}_{0}, \mathbf{y}_{0} ; \mathbf{g}_{w}, \mathbf{g}_{p}\right) \mathbf{g}_{p}\right)+\mathbf{w}_{g}^{*}\left(T E^{D}\left(\mathbf{x}_{0}, \mathbf{y}_{0} ; \mathbf{g}_{w}, \mathbf{g}_{p}\right) \mathbf{g}_{w}\right)}{\mathbf{p}_{g}^{*} \mathbf{y}_{0}-\mathbf{w}_{g}^{*} \mathbf{x}_{0}^{v}}\right\} .
$$

The following proposition shows that the optimal solution to this problem obtains the McFadden gauge function (8).

Proposition 1. The measurement direction underlying the McFadden gauge function (8) minimizes the short run profit inefficiency of the evaluated firm. In other words, setting $\left(\mathbf{g}_{w}, \mathbf{g}_{p}\right)=\left(-\mathbf{x}_{0}^{v}, \mathbf{y}_{0}\right)$ gives the optimal solution to problem (12).

Proof. By substituting $\mathbf{g}=\left(-\mathbf{x}_{0}^{v}, \mathbf{y}\right)$ in (12), the profit inefficiency is:

$$
P E^{P}\left(\mathbf{x}_{0}, \mathbf{y}_{0} ; \mathbf{w}_{\mathrm{g}}^{*}, \mathbf{p}_{\mathrm{g}}^{*}\right)=T E^{D}\left(\mathbf{x}_{0}, \mathbf{y}_{0} ;-\mathbf{x}_{0}^{v}, \mathbf{y}_{0}\right)
$$

Next consider an arbitrary direction $\mathbf{g}$. The technically efficient production plan in the direction $\mathbf{g}$ is (10), which is profit maximizing for the price system $\left(\mathbf{w}_{g}^{*} \mathbf{p}_{g}^{*}\right)$ defined as the shadow price vector given by the normal of the plane that supports $T$ at that production plan. We thus always have

$$
\begin{aligned}
& \pi\left(\mathbf{x}_{0}^{f} ; \mathbf{w}_{g}^{*}, \mathbf{p}_{g}^{*}\right)=\mathbf{p}_{g}^{*}\left(\mathbf{y}_{0}+T E^{D}\left(\mathbf{x}_{0}, \mathbf{y}_{0} ; \mathbf{g}_{w}, \mathbf{g}_{p}\right) \mathbf{g}_{p}\right)-\mathbf{w}_{g}^{*}\left(\mathbf{x}_{0}^{v}-T E^{D}\left(\mathbf{x}_{0}, \mathbf{y}_{0} ; \mathbf{g}_{w}, \mathbf{g}_{p}\right) \mathbf{g}_{w}\right) \geq \\
& \mathbf{p}_{g}^{*}\left(\mathbf{y}_{0}+T E^{D}\left(\mathbf{x}_{0}, \mathbf{y}_{0} ;-\mathbf{x}_{0}^{v}, \mathbf{y}_{0}\right) \mathbf{y}_{0}\right)-\mathbf{w}_{g}^{*}\left(\mathbf{x}_{0}^{v}+T E^{D}\left(\mathbf{x}_{0}, \mathbf{y}_{0} ;-\mathbf{x}_{0}^{v}, \mathbf{y}_{0}\right) \mathbf{x}_{0}^{v}\right) \quad \forall \mathbf{g} .
\end{aligned}
$$

Some straightforward algebraic manipulations consequently entail:

$$
T E^{D}\left(\mathbf{x}_{0}, \mathbf{y}_{0} ;-\mathbf{x}_{0}^{v}, \mathbf{y}_{0}\right) \leq \frac{\mathbf{p}_{g}^{*}\left(T E^{D}\left(\mathbf{x}_{0}, \mathbf{y}_{0} ; \mathbf{g}_{w}, \mathbf{g}_{p}\right) \mathbf{g}_{p}\right)+\mathbf{w}_{g}^{*}\left(T E^{D}\left(\mathbf{x}_{0}, \mathbf{y}_{0} ; \mathbf{g}_{w}, \mathbf{g}_{p}\right) \mathbf{g}_{w}\right)}{\mathbf{p}_{g}^{*} \mathbf{y}_{0}-\mathbf{w}_{g}^{*} \mathbf{x}_{0}^{v}} \quad \forall \mathbf{g},
$$

which implies

$$
T E^{D}\left(\mathbf{x}_{0}, \mathbf{y}_{0} ;-\mathbf{x}_{0}^{v}, \mathbf{y}_{0}\right)=\inf _{\mathbf{g}}\left\{\frac{\mathbf{p}_{g}^{*}\left(T E^{D}\left(\mathbf{x}_{0}, \mathbf{y}_{0} ; \mathbf{g}_{w}, \mathbf{g}_{p}\right) \mathbf{g}_{p}\right)+\mathbf{w}_{g}^{*}\left(T E^{D}\left(\mathbf{x}_{0}, \mathbf{y}_{0} ; \mathbf{g}_{w}, \mathbf{g}_{p}\right) \mathbf{g}_{w}\right)}{\mathbf{p}_{g}^{*} \mathbf{y}_{0}-\mathbf{w}_{g}^{*} \mathbf{x}_{0}^{v}}\right\}
$$


For completeness, it is worth noting that the optimum to problem (12) is not unique. The directional distance function is homogenous of degree -1 in $\mathbf{g}$ (e.g. Chambers et al., 1998), so any vector collinear to $\mathbf{g}=\left(-\mathbf{x}_{0}^{v}, \mathbf{y}_{0}\right)$ will yield the same result: the modulus of the vector is arbitrary. In other words, all alternate optima are scalar multiples of vector $\mathbf{g}=\left(-\mathbf{x}_{0}^{v}, \mathbf{y}_{0}\right)$.

\section{Summary}

We have examined the measurement of economic and technical efficiency in the framework of short run profit maximizing behavior, drawing special attention on the corresponding duality relationship. First, we have reviewed alternative profit efficiency indices proposed in the literature, and identified Varian's (1990) percentage profit efficiency measure as our preferred alternative for evaluating short run profit efficiency. Next, we have shown that the McFadden gauge function provides a natural dual for this measure. Our argument is based on the two criteria suggested by Russell (1985): the McFadden gauge function (i) can be represented as Varian's profit efficiency measure evaluated at shadow prices, and (ii) provides an upper bound for that profit efficiency measure that applies for any possible system of the market prices. Finally, we have considered the choice of measurement direction from the perspective of the evaluated firm within the general directional distance function framework of Chambers et al. $(1996,1998)$. We have shown that the McFadden gauge function is the optimal measurement direction for the evaluated firm in the sense that the corresponding reference production plan and associated shadow prices implies minimal profit inefficiency as calculated with respect to that reference production plan.

Our results provide the profit efficiency counterpart for Russell's (1985) cost efficiency argument in favor of the Farrell input efficiency measure. While Russell asserts that the Farrell input efficiency measure is the natural dual technical efficiency measure under cost minimizing behavior, we find that similar arguments institute the modified McFadden gauge function as the natural dual to the measure of economic efficiency under short run profit maximizing behavior. These theoretical insights are of direct relevance for the empirical research on economic and technical efficiency in general and firm-level short run profit efficiency in particular (see Blancard et al. (2006) for a recent contribution). Specifically, our modified McFadden gauge function provides a natural direction vector for efficiency measurement, which can be directly employed in empirical estimation of economic and technical efficiency. 


\section{References}

Chambers, R., Y. Chung, and R. Färe (1996). "Benefit and distance functions", Journal of Economic Theory, 70(2), pp.407-419.

Chambers R., Chung Y., and Färe R., (1998). "Profit, directional distance functions, and Nerlovian efficiency", Journal of Optimization Theory and Applications, 98(2), pp.351-364.

Blancard S., J.P. Boussemart, W. Briec, and K. Kerstens (2006). "Short- and LongRun Credit Constraints in French Agricultue: A Directional Distance Function Framework Using Expenditure-Constrained Profit Functions", American Journal of Agricultural Economics, 88(2), pp.351-364.

Boussemart, J.P., W. Briec, N. Peypoch, and C. Tavera (2008). " $\alpha$-returns to scale in multi-outputs production technologies", European Journal of Operational Research, forthcoming.

Debreu G. (1951). "The coefficient of resource utilization", Econometrica, 19(3), pp.273-292.

Domazlicky, B.R., and Weber, W.L. (2004). "Does Environmental Protection Lead to Slower Productivity Growth in the Chemical Industry?", Environmental and Resource Economics 28(3), pp.301-324.

Färe R., and Grosskopf S. (2000). "Notes on some inequalities in economics", Economic Theory 15, pp.227-233.

Färe R., Grosskopf S., and Pasurka, C.A. Jr. (2001). "Accounting for air pollution emissions in measures of state manufacturing productivity growth", Journal of Regional Science 41(3), pp.381-409.

Färe R., and Primont, D. (1995). Multi-output production and duality: theory and applications, Kluwer Academic Publishers; Boston, London, Dordrecht.

Farrell, M. (1957). "The measurement of productive efficiency", Journal of the Royal Statistical Society, Series A: General, 120(3), pp.253-281.

Kuosmanen, T. (2003). "Duality Theory of Non-convex Technologies", Journal of Productivity Analysis 20, pp.273-304.

Luenberger D.G. (1992). "Benefit Functions and Duality", Journal of Mathematical Economics, 21, pp.461-481.

McFadden D. (1978). Cost, revenue, and profit functions, Production economics: a dual approach to theory and applications; Edited by M. Fuss and D. McFadden, North Holland, Amsterdam, Holland, Vol 1, pp.1-110.

Nerlove M. (1965). Estimation and identification of Cobb-Douglas production functions, Rand McNally Company, Chicago, Illinois.

Russell R. (1985). "Measures of technical efficiency", Journal of Economic Theory 35 (1), pp.109-126.

Salnykov M., Zeleniuk V. (2005). "On the commensurability of directional distance functions", Dicussion paper 0517, Institut de Statistique, Université Catholique de Louvain.

Shephard R.W. (1953). Cost and production functions, Princeton University Press, Princeton, New Jersey.

Shephard R.W. (1970). Theory of cost and production functions, Princeton University Press, Princeton, New Jersey.

Varian H.R. (1990). "Goodness-of-fit in demand analysis", Journal of Econometrics, 46, pp.125-140. 